\title{
A fixed point theorem for biased maps satisfying an implicit relation
}

\author{
Awnish Singh and Koti Prasad \\ Department of Pure \& Applied Mathematics, Guru Ghasidas Vishwavidyalaya, Bilaspur, India \\ Received: 18 April 2018, Accepted: 31 December 2018 \\ Published online: 17 March 2019.
}

\begin{abstract}
In this paper, we establish a common fixed point theorem for four self mappings satisfying an implicit relation, via weakly biased maps in metric space.
\end{abstract}

Keywords: Implicit relation, contraction, coincidence point, weakly biased map, common fixed point, metric space.

\section{Introduction}

Finding fixed point through implicit functions is an interesting concept. In 1997, Popa [11,12] introduced implicit functions without contraction conditions to prove fixed point theorems in metric spaces. Implicit function useful to several contraction conditions simultaneously to find known as well as unknown contraction conditions. Latter, S. Sharma and B. Deshpande [14], established an implicit relation for compatible mappings in Banach spaces. Subsequently, Javid Ali and Imdad [4] define implicit function of contraction conditions in metric space to prove a general common fixed point theorem of weakly compatible mappings satisfying the common property (E.A.). In 2006 , I. Altun, H.A. Hancer and D. Turkoglu [1], proved a fixed point theorem for multivalued mapping satisfying an implicit relation on metrically convex metric spaces. Many authors have proved common fixed point theorems under implicit relation conditions for this we refer $[2,3,6,15]$.

\section{Implicit relations}

Let $\Phi$ be the set of all real continuous functions $\phi: \mathbb{R}_{+}^{5} \rightarrow \mathbb{R}_{+}$satisfying the following conditions;

$$
\begin{aligned}
& \phi_{1}: \text { is non-increasing on each variable } \\
& \phi_{2}: \text { there exists } k \in(0,1) \text { such that for every } u, v \geq 0 \text { with } \\
& \phi(u, v, v, u, u) \leq 0, \text { or } \phi\left(u, v, v, u, \frac{1}{2}(u+v)\right) \leq 0, \text { for all } u \leq k v . \\
& \phi_{3}: \text { for every } u, v \geq 0 \text { and } a \in(0,2] \text { with } \\
& \phi_{3 a}: \phi(u, u, a u, 0, u)>0, \\
& \phi_{3 b}: \phi(u, u, 0, a u, u)>0, \\
& \phi_{3 c}: \phi(0, u, u, 0, a u)>0,
\end{aligned}
$$

\footnotetext{
*Corresponding author e-mail: knvp71@yahoo.co.in, awnish.singh85@gmail.com
} 


$$
\begin{aligned}
& \phi_{4}: \phi\left(u, 0,0, u, \frac{1}{2} u\right)>0 \text { or } \phi\left(0, u, u, 0, \frac{1}{2} u\right)>0 \\
& \phi_{5}: \phi(u, u, 0,0, u)>0, \text { for all } u>0 .
\end{aligned}
$$

Example 1. Define $F\left(t_{1}, t_{2}, \ldots t_{5}\right): \mathbb{R}_{+}^{5} \rightarrow \mathbb{R}_{+}$as

$$
F\left(t_{1}, t_{2}, \ldots t_{5}\right)=t_{1}-k \max \left\{t_{2}, t_{3}, t_{4}, t_{5}\right\}
$$

where $k \in[0,1)$, clearly $F \in \Phi$.

Example 2. Define $F\left(t_{1}, t_{2}, \ldots t_{5}\right): \mathbb{R}_{+}^{5} \rightarrow \mathbb{R}_{+}$as

$$
F\left(t_{1}, t_{2}, \ldots t_{5}\right)=t_{1}-k \max \left\{t_{2} \cdot t_{3}, t_{3} \cdot t_{4}, t_{4} \cdot t_{5}\right\}
$$

where $k \in[0,1)$, clearly $F \in \Phi$.

Example 3. Define $F\left(t_{1}, t_{2}, \ldots t_{5}\right): \mathbb{R}_{+}^{5} \rightarrow \mathbb{R}_{+}$as

$$
F\left(t_{1}, t_{2}, \ldots t_{5}\right)=t_{1}-\alpha\left(t_{3}, t_{4}\right)
$$

where $\alpha \in\left[0, \frac{1}{2}\right)$, clearly $F \in \Phi$.

Example 4. Define $F\left(t_{1}, t_{2}, \ldots t_{5}\right): \mathbb{R}_{+}^{5} \rightarrow \mathbb{R}_{+}$as

$$
F\left(t_{1}, t_{2}, \ldots t_{5}\right)=t_{1}-\alpha\left(t_{4}, t_{5}\right)
$$

where $\alpha \in\left[0, \frac{1}{2}\right)$, clearly $F \in \Phi$.

Example 5. Define $F\left(t_{1}, t_{2}, \ldots t_{5}\right): \mathbb{R}_{+}^{5} \rightarrow \mathbb{R}_{+}$as

$$
F\left(t_{1}, t_{2}, \ldots t_{5}\right)=t_{1}^{2}-\alpha t_{1} \cdot t_{2}-\beta t_{3} \cdot t_{4}-\gamma t_{4} \cdot t_{5},
$$

where $\alpha, \beta, \gamma \geq 0$ and $\alpha+\beta+\gamma<1$, clearly $F \in \Phi$.

Example 6. Define $F\left(t_{1}, t_{2}, \ldots t_{5}\right): \mathbb{R}_{+}^{5} \rightarrow \mathbb{R}_{+}$as

$$
F\left(t_{1}, t_{2}, \ldots t_{5}\right)=t_{1}-\alpha \max \left\{t_{2}, t_{3}\right\}-\beta \max \left\{t_{2}+t_{3}, t_{4}+t_{5}\right\},
$$

where $\alpha, \beta \geq 0$ and $\alpha+2 \beta<1$, clearly $F \in \Phi$.

In 1995, G.Jungck and H.K. Pathak [8], introduced the definitions of biased maps and weakly biased maps.

Definition 1. [8] Let $A$ and $S$ be self-maps of a metric space $(X, d)$. The pair $(A, S)$ is $S$-biased iff whenever $\left\{x_{n}\right\}$ is a sequence in $X$ and $A x_{n}, S x_{n} \rightarrow t \in X$, then

$$
\alpha d\left(S A x_{n}, S x_{n}\right) \leq \alpha d\left(A S x_{n}, A x_{n}\right) \text { if } \alpha=\lim \inf \text { and if } \alpha=\lim \sup .
$$

Definition 2. [8] Let $A$ and $S$ be self-maps of a metric space $(X, d)$. The pair $(A, S)$ is weakly S-biased iff Ap $=S p$ implies

$$
d(S A p, S p) \leq d(A S p, A p)
$$

Definition 3. [10] Let $A$ and $T$ be selfmaps of a set $X$. If $A x=T x=w$ (say), $w \in X$, for some $x$ in $X$, then $x$ is called a coincidence point of $A$ and $T$ and $w$ is called point of coincidence of $A$ and $T$.

Definition 4. Let $A$ and $T$ be selfmaps of a set $X$, then the pair $(A, T)$ is said to 
(i) be compatible [7] if $\lim _{n \rightarrow \infty} d\left(A T x_{n}, T A x_{n}\right)=0$, whenever $\left\{x_{n}\right\}$ is a sequence in Xsuch that $\lim _{n \rightarrow \infty} A x_{n}=\lim _{n \rightarrow \infty} T x_{n}=t$, for some $t \in X$.

(ii) be weakly compatible [9] if TAx $=$ ATx whenever $A x=T x, x \in X$.

(iii) be occasionally weakly compatible (owc) [16] if TAx $=A T x$ for some $x \in C(A, T)$, where $C(A, T)$ is the set of coincidence points of $A$ and $T$.

The relations between the above definitions are as follows.

(i) Every compatible pair is weakly compatible but its converse need not be true[9].

(ii) Every weakly compatible pair is occasionally weakly compatible but its converse need not be true[16].

In this paper we established a common fixed point theorem by using biased map through a new type implicit function.

\section{Main result}

Our main result is following. Let $A, B, S$ and $T$ be a selfmaps of metric space $X$ satisfying the following conditions:

$$
\phi\left(d(A x, B y), d(S x, T y), d(S x, A x), d(T y, B y), \frac{1}{2}[d(S x, B y)+d(A x, T y)]\right) \leq 0
$$

for all $x, y \in X$, where $\phi \in \Phi$ and $A(X) \subset T(X)$ and $B(X) \subset S(X)$.

for an arbitrary point $x_{0} \in X$ there exists a point $x_{1} \in X$ such that $A x_{0}=T x_{1}$. Since $B(X) \subset S(X)$ for this point $x_{1} \in X$, we can choose a point $x_{2} \in X$ such that $B x_{1}=S x_{2}$ and so on, we can define a sequence $\left\{y_{n}\right\}$ in $X$ such that

$$
y_{2 n}=A x_{2 n}=T x_{2 n+1} \text { and } y_{2 n+1}=B x_{2 n+1}=S x_{2 n+2} \text { for every } n=0,1,2 \ldots \ldots
$$

Lemma 1. Let $(X, d)$ be a metric space and $A, B, S$ and $T$ be a selfmaps of $X$ satisfying the following conditions (1) and (2). Then the sequence $\left\{y_{n}\right\}$ is Cauchy sequence in $X$.

Proof. On taking $x=x_{2 n}$ and $y=x_{2 n+1}$ in (1), then we obtain

$$
\phi\left(d\left(A x_{2 n}, B x_{2 n+1}\right), d\left(S x_{2 n}, T x_{2 n+1}\right), d\left(S x_{2 n}, A x_{2 n}\right), d\left(T x_{2 n+1}, B x_{2 n+1}\right), \frac{1}{2}\left[d\left(S x_{2 n}, B x_{2 n+1}\right)+d\left(A x_{2 n}, T x_{2 n+1}\right)\right]\right) \leq 0 .
$$

By using (2), we have

$$
\phi\left(d\left(y_{2 n}, y_{2 n+1}\right), d\left(y_{2 n-1}, y_{2 n}\right), d\left(y_{2 n-1}, y_{2 n}\right), d\left(y_{2 n}, y_{2 n+1}\right), \frac{1}{2}\left[d\left(y_{2 n-1}, y_{2 n+1}\right)+d\left(y_{2 n}, y_{2 n}\right)\right]\right) \leq 0 .
$$

Let us consider $\alpha_{2 n}=d\left(y_{2 n}, y_{2 n+1}\right)$ and $\alpha_{2 n-1}=d\left(y_{2 n-1}, y_{2 n}\right)$. Then, by using triangular inequality, the property $\phi_{1}$ and $\phi_{2}$ and (3), we get

$$
\begin{gathered}
\phi\left(\alpha_{2 n}, \alpha_{2 n-1}, \alpha_{2 n-1}, \alpha_{2 n}, \frac{1}{2}\left[\alpha_{2 n}+\alpha_{2 n-1}\right]\right) \leq 0 \\
\alpha_{2 n} \leq k \alpha_{2 n-1}<\alpha_{2 n-1}
\end{gathered}
$$

Similarly, we can show that $\alpha_{2 n+1}<\alpha_{2 n}$, therefore

$$
\alpha_{n}<\alpha_{n+1} \forall n
$$

Therefore $\left\{\alpha_{n}\right\}=\left\{d\left(y_{n}, y_{n+1}\right)\right\}$ is decreasing sequence and hence

$$
\lim _{n \rightarrow \infty} d\left(y_{n}, y_{n+1}\right)=0
$$


Now, we have to show that the sequence $\left\{y_{n}\right\}$ is a Cauchy in $X$, for this it is sufficient to show that $\left\{y_{2 n}\right\}$ is a Cauchy sequence. If possible $\left\{y_{2 n}\right\}$ is not a Cauchy sequence, then $\exists \varepsilon>0$ s.t. for each even integer $k, \exists$ an even integer $2 n(k)$ and $2 m(k)$ with $2 m(k)>2 n(k)>k$ s.t.

$$
d\left(y_{2 n(k)}, y_{2 m(k)}\right) \geq \varepsilon
$$

for each even integer $k$, let $2 m(k)$ be the least positive integer exceeding $2 n(k)$ satisfying (6) then we have $d\left(y_{2 n(k)}, y_{2 m(k)-2}\right)<\varepsilon$ and triangle inequality

$$
\begin{aligned}
\varepsilon \leq d\left(y_{2 n(k)}, y_{2 m(k)}\right) & \leq d\left(y_{2 n(k)}, y_{2 m(k)-2}\right)+d\left(y_{2 m(k)-2}, y_{2 m(k)-1}\right)+d\left(y_{2 m(k)-1}, y_{2 m(k)}\right) \\
& <\varepsilon+d\left(y_{2 m(k)-2}, y_{2 m(k)-1}\right)+d\left(y_{2 m(k)-1}, y_{2 m(k)}\right) .
\end{aligned}
$$

On taking $\lim _{k \rightarrow \infty}$, we have

$$
\lim _{k \rightarrow \infty} d\left(y_{2 n(k)}, y_{2 m(k)}\right)=\varepsilon
$$

By using triangular inequality, we have

$$
\left|d\left(y_{2 n(k)}, y_{2 m(k+1)}\right)-d\left(y_{2 n(k)}, y_{2 m(k)}\right)\right| \leq d\left(y_{2 n(k)}, y_{2 n(k)+1}\right) .
$$

On taking limits as $k \rightarrow \infty$, we get

$$
\lim _{k \rightarrow \infty} d\left(y_{2 n(k)}, y_{2 m(k+1)}\right)=\varepsilon
$$

Again using triangular inequality, we have

$$
\left|d\left(y_{2 n(k)-1}, y_{2 m(k)}\right)-d\left(y_{2 n(k)}, y_{2 m(k)}\right)\right| \leq d\left(y_{2 n(k)}, y_{2 n(k)-1}\right) .
$$

Applying limits as $k \rightarrow \infty$, we get

$$
\lim _{k \rightarrow \infty} d\left(y_{2 n(k)-1}, y_{2 m(k)}\right)=\varepsilon .
$$

And Again using triangular inequality, we have

$$
\left|d\left(y_{2 n(k)-1}, y_{2 m(k)+1}\right)-d\left(y_{2 n(k)-1}, y_{2 m(k)}\right)\right| \leq d\left(y_{2 m(k)}, y_{2 m(k)+1}\right),
$$

Applying limits as $k \rightarrow \infty$, we get

$$
\lim _{k \rightarrow \infty} d\left(y_{2 n(k)-1}, y_{2 m(k)+1}\right)=\varepsilon .
$$

On taking $x=x_{2 n(k)}$ and $y=x_{2 m(k)+1}$ in (1), we obtain

$$
\begin{gathered}
\phi\left(d\left(A x_{2 n(k)}, B x_{2 m(k)+1}\right), d\left(S x_{2 n(k)}, T x_{2 m(k)+1}\right), d\left(S x_{2 n(k)}, A x_{2 n(k)}\right), d\left(T x_{2 m(k)+1}, B x_{2 m(k)+1}\right),\right. \\
\left.\frac{1}{2}\left[d\left(S x_{2 n(k)}, B x_{2 m(k)+1}\right)+d\left(A x_{2 n(k)}, T x_{2 m(k)+1}\right)\right]\right) \leq 0
\end{gathered}
$$

by using (2), we get

$$
\begin{gathered}
\phi\left(d\left(y_{2 n(k)}, y_{2 m(k)+1}\right), d\left(y_{2 n(k)-1}, y_{2 m(k)}\right), d\left(y_{2 n(k)-1}, y_{2 n(k)}\right), d\left(y_{2 m(k)}, y_{2 m(k)+1}\right),\right. \\
\left.\frac{1}{2}\left[d\left(y_{2 n(k)-1}, y_{2 m(k)+1}\right)+d\left(y_{2 n(k)}, y_{2 m(k)}\right)\right]\right) \leq 0,
\end{gathered}
$$

On taking $\lim _{k \rightarrow \infty}$ and using and (7)-(10), we have

$$
\phi(\varepsilon, \varepsilon, 0,0, \varepsilon) \leq 0
$$


a contradiction, by the property $\phi_{5}$. Therefore $\left\{y_{2 n}\right\}$ is a Cauchy sequence. Consequently $\left\{y_{2 n+1}\right\}$ is a Cauchy sequence. Hence $\left\{y_{n}\right\}$ is a Cauchy sequence in $X$.

Proposition 1. Let $(X, d)$ be a metric space and $A, B, S$ and $T$ be a selfmaps of $X$ satisfying inequality (1) and suppose that $R=\{x \in X: A x=S x\}$ and $P=\{x \in X: B x=T x\}$. Then, either

(a) $A(X) \subset T(X)$ and $R \neq \phi \Rightarrow P \neq \phi$ holds or

(b) $B(X) \subset S(X)$ and $P \neq \phi \Rightarrow R \neq \phi$ holds.

Furthermore, A, B, S and T have a common coincidence point in $X$.

Proof. Assume that ( $a$ ) holds. Let $R$ is non empty i.e $A t=S t, t \in R$. Since $A(X) \subset T(X)$, there is a point $w \in X$ such that $A t=T w$. Therefore

$$
A t=S t=T w
$$

Now, we show that $B w=T w$, assume that $B w \neq T w$, on taking $x=t$ and $y=w$ in (1), we get

$$
\phi\left(d(A t, B w), d(S t, T w), d(S t, A t), d(T w, B w), \frac{1}{2}[d(S t, B w)+d(A t, T w)]\right) \leq 0 .
$$

From (11), we get

$$
\phi\left(d(T w, B w), d(T w, T w), d(S t, A t), d(T w, B w), \frac{1}{2}[d(T w, B w)+d(T w, T w)]\right) \leq 0,
$$

implies that

$$
\phi\left(d(T w, B w), 0,0, d(T w, B w), \frac{1}{2}[d(T w, B w)]\right) \leq 0,
$$

a contradiction, by the property of $\phi_{4}$. Thus

$$
B w=T w \text { implies that } P \text { is non empty }
$$

Hence $A, B, S$ and $T$ have a common coincidence point in $X$. Similarly, the procedure of proof is same lines if (b) holds.

Proposition 2. Let $A, B$, S and $T$ self maps on metric space $X$. If the pairs $(A, S)$ and $(B, T)$ are have a common coincidence point. Then $A, B, S$ and $T$ have unique common fixed point in $X$, provided the pairs $(A, S)$ is $S$-weakly biased map and $(B, T)$ is B-weakly biased map.

Proof. Let $A, B, S$ and $T$ have a common coincidence point in $X$. i.e

$$
A t=S t=T w=B w=r .(\text { say }) \text { for some } t, w \in X
$$

First, we show that $r$ is fixed point of $A$. Suppose $A r \neq r$, choose $x=r$ and $y=w$ in (1), we have

$$
\phi\left(d(A r, B w), d(S r, T w), d(S r, A r), d(T w, B w), \frac{1}{2}[d(S r, B w)+d(A r, T w)]\right) \leq 0 .
$$

From (12), we obtain that

$$
\phi\left(d(A r, r), d(S r, r), d(S r, r)+d(A r, r), d(r, r), \frac{1}{2}[d(S r, r)+d(A r, r)]\right) \leq 0 .
$$

Since the pair $(A, S)$ is $S$-weakly biased then from (12), we have

$$
A t=S t \Rightarrow d(S A t, S t) \leq d(A S t, A t) d(S r, r) \leq d(A r, r) .
$$


Therefore, by using (13)-(14), we get

$$
\phi(d(A r, r), d(A r, r), 2 d(A r, r), 0, d(A r, r)) \leq 0
$$

a contradiction, by the property of $\phi_{3 a}$. Hence

$$
A r=r
$$

Thus, from (14) and (15), we get

$$
A r=S r=r
$$

Now, finally we show that $r$ is a common fixed point of $B$ and $T$. Since the pair $(B, T)$ is $B$-weakly biased map then from (12), we have

$$
B w=T w \Rightarrow d(B T w, B w) \leq d(T B w, T w) \Rightarrow d(B r, r) \leq d(T r, r)
$$

Now, we show that $T r=r$, suppose that $T r \neq r$, choose $x=r$ and $y=r$ in (1), we get

$$
\phi\left(d(A r, B r), d(S r, T r), d(S r, A r), d(\operatorname{Tr}, B r), \frac{1}{2}[d(S r, B r)+d(A r, T r)]\right) \leq 0 .
$$

By using (16), we have

$$
\phi\left(d(r, B r), d(r, T r), 0, d(\operatorname{Tr}, B r), \frac{1}{2}[d(r, B r)+d(r, T r)]\right) \leq 0 .
$$

By using triangle inequality and (17) and property of $\phi_{1}$, we get

$$
\phi(d(r, T r), d(r, T r), 0,2 d(r, T r), d(r, T r)) \leq 0
$$

a contradiction, by the property of $\phi_{3 b}$. Thus

$$
\operatorname{Tr}=r \Longrightarrow B r=r .(b y(17))
$$

Hence $A, B, S$ and $T$ have common fixed point in $X$. Uniqueness is easily verify that by traditional method.

Theorem 1. Let $(X, d)$ be a metric space and $A, B, S$ and $T$ be a selfmaps of $X$ satisfying the inequality $(1) ; A(X) \subset T(X)$ and $B(X) \subset S(X)$, one of the ranges $A(X), B(X), S(X)$ and $T(X)$ are a complete subspace of $X$, the pairs $(A, S)$ is $S$-weakly biased and $(B, T)$ is $B$-weakly biased mapping. Then $A, B, S$ and $T$ have a unique common fixed point in $X$.

Proof. From Lemma 1. the sequence $\left\{y_{n}\right\}$ is Cauchy in $X$. Assume that $A(X)$ is a complete therefore,

$$
\lim _{n \rightarrow \infty} y_{2 n}=\lim _{n \rightarrow \infty} A x_{2 n}=\lim _{n \rightarrow \infty} T x_{2 n+1}=z .(\in X)
$$

Since $\left\{y_{n}\right\}$ is a Cauchy, it follows that $\lim _{n \rightarrow \infty} y_{n}=z$. Thus $A x_{2 n}, B x_{2 n+1}, S x_{2 n+2}, T x_{2 n+1}$ are converges to a point $z$ in $X$.

$$
\lim _{n \rightarrow \infty} A x_{2 n}=\lim _{n \rightarrow \infty} B x_{2 n+1}=\lim _{n \rightarrow \infty} S x_{2 n+2}=\lim _{n \rightarrow \infty} T x_{2 n+1}=z(=A u) .
$$

Since, $A(X) \subset T(X), \exists v \in X$ s.t.

$$
A u=T v \cdot(=z)
$$

Now, we show that $A u \neq S u$, choose $x=u$ and $y=x_{2 n+1}$ in (1), we obtain

$$
\begin{gathered}
\phi\left(d\left(A u, B x_{2 n+1}\right), d\left(S u, T x_{2 n+1}\right), d(S u, A u), d\left(T x_{2 n+1}, B x_{2 n+1}\right),\right. \\
\left.\frac{1}{2}\left[d\left(S u, B x_{2 n+1}\right)+d\left(A u, T x_{2 n+1}\right)\right]\right) \leq 0 .
\end{gathered}
$$


On taking $\lim _{n \rightarrow \infty}$ and using (21), we get

$$
\phi\left(d(A u, z), d(S u, z), d(S u, A u), d(z, z), \frac{1}{2}[d(S u, z)+d(A u, z)]\right) \leq 0,
$$

By, property $\phi_{1}$ and (21), we get

$$
\phi\left(0, d(S u, z), d(S u, z), 0, \frac{1}{2} d(S u, z)\right) \leq 0,
$$

a contradiction, by the property of $\phi_{4}$. Hence

$$
S u=z, \Rightarrow A u=S u=z
$$

Then from Proposition 1. and Proposition 2, we get $z$ is a common fixed point of $A, B, S$ and $T$ in $X$. Uniqueness follows from inequality (1) easily.

Corollary 1. Let $(X, d)$ be a complete metric space and $A, B, S$ and $T$ be a selfmaps of $X$ satisfying the following conditions;

$$
\phi\left(d(A x, B y), d(S x, T y), d(S x, A x), d(T y, B y), \frac{1}{2}[d(S x, B y)+d(A x, T y)]\right) \leq 0
$$

for all $x, y \in X$, where $\phi \in \Phi . A(X) \subset T(X)$ and $B(X) \subset S(X)$, one of $A, B, S, T$ is continuous, the pairs $(A, S)$ and $(B, T)$ are compatible maps. Then $A, B, S$ and $T$ have a unique common fixed point.

Corollary 2. Let $(X, d)$ be a complete metric space and $A, B, S$ and $T$ be a selfmaps of $X$ satisfying the following conditions;

$$
\phi\left(d(A x, B y), d(S x, T y), d(S x, A x), d(T y, B y), \frac{1}{2}[d(S x, B y)+d(A x, T y)]\right) \leq 0
$$

for all $x, y \in X$, where $\phi \in \Phi . A(X) \subset T(X)$ and $B(X) \subset S(X)$, one of $A, B, S, T$ is continuous, $(A, S)$ and $(B, T)$ are weakly compatible maps. Then $A, B, S$ and $T$ have a unique common fixed point.

Corollary 3. Let $(X, d)$ be a complete metric space and A, B, S and T be a selfmaps of X satisfying the following conditions;

$$
\phi\left(d(A x, B y), d(S x, T y), d(S x, A x), d(T y, B y), \frac{1}{2}[d(S x, B y)+d(A x, T y)]\right) \leq 0
$$

for all $x, y \in X$, where $\phi \in \Phi . A(X) \subset T(X)$ and $B(X) \subset S(X)$, one of $A, B, S, T$ is continuous, $(A, S)$ and $(B, T)$ are occasionally weakly compatible maps. Then $A, B, S$ and $T$ have a unique common fixed point.

The following example is support of our main theorem and not to applicable to Corollary 1 and Corollary 2.

Example 7. Let $X=[0,1]$ be endowed with the Euclidean metric $d(x, y)=|x-y|$, and $A, B, S$ and $T$ be self maps in $X$,

$$
\begin{aligned}
& A(X)=\left\{\begin{array}{l}
0 \text { if } x \in(0,1) \\
\frac{1}{4} \text { if } x=1
\end{array} \quad B(X)=\left\{\begin{array}{ll}
0 \text { for all } x \\
S(X)= \begin{cases}0 & \text { if } x=0 \\
1 & \text { if } x \neq 0\end{cases}
\end{array} T(X)= \begin{cases}0 & \text { if } x=0 \\
1-x & \text { if } x \neq 0\end{cases} \right.\right.
\end{aligned}
$$

$A(X)=\left\{0, \frac{1}{4}\right\} \subset T(X)=[0,1)$ and $B(X)=\{0\} \subset S(X)=\{0,1\}$, clearly $A(X)$ is a complete subspace of X. Finally, we have to verify that condition (1) holds, our $\Phi$ is the set of all real continuous function $\phi\left(t_{1}, t_{2}, \ldots t_{5}\right): \mathbb{R}_{+}^{5} \rightarrow \mathbb{R}_{+}$satisfying the conditions given in above. Define by $F\left(t_{1}, t_{2}, \ldots t_{5}\right): \mathbb{R}_{+}^{5} \rightarrow \mathbb{R}_{+}$as

$$
F\left(t_{1}, t_{2}, \ldots t_{5}\right)=t_{1}-k \max \left\{t_{2}, t_{3}, t_{4}, t_{5}\right\}
$$


where $k \in[0,1)$, we verify $F \in \Phi$. $\left(\phi_{1}\right)$ : is trivial. $\left(\phi_{2}\right)$ : Let $u>0, \phi(u, v, v, u, u)=u-k \max \{v, v, u, u\} \leq 0$. If $u \geq v$ then $u \leq k u<u$, a contradiction. Thus $u<v$ and $u \leq k v$, where $k \in(0,1)$. $\left(\phi_{3 a}\right)$ : $\phi(u, u, a u, 0, u)=u-k \max \{u, a u, 0, u\}=(1-k) u>0$, for all $u>0$. $\left(\phi_{3 b}\right)$ : Similarly $\phi(u, u, 0, a u, u)>0 .\left(\phi_{3 c}\right)$ : $\phi(0, u, u, 0, a u)>0 .\left(\phi_{4}\right): \phi\left(u, 0,0, u, \frac{1}{2} u\right)=u-k \max \left\{u, \frac{1}{2} u\right\}=\left(1-\frac{k}{2}\right) u>0$, for all $u>0 .\left(\phi_{5}\right): \phi(u, u, 0,0, u)>0$ for all $u>0$, satisfying all the conditions of implicit relation.

Finally, we have to show that $\phi$ is satisfying our inequality (1). Only two cases are arises i.e

Case I. If $x=1$ and $y=0$

$$
\frac{1}{4} \leq k \max \left\{1, \frac{3}{4}, 0, \frac{5}{8}\right\}
$$

Case II. If $x=1$ and $y \neq 0$

$$
\frac{1}{4} \leq k \max \left\{y, \frac{3}{4}, 1-y, \frac{1+4 y}{8}\right\} .
$$

Rest of all possible cases vanishes. It is easy to observe that theorem 1 required conditions are holds and also 0 is the unique common fixed point of $\mathrm{A}, \mathrm{B}, \mathrm{S}$ and T. At the fixed point the pairs $(A, S)$ and $(B, T)$ satisfying S-weakly biased and B-weakly biased.

Remark. The interesting note is that the above example the pair $(B, T)$ is not compatible, there exist a sequence $\left\{x_{n}\right\}$ in X such that $x_{n}=1-\frac{1}{n}, n>1$, then $\lim _{n \rightarrow \infty} B x_{n}=0$ and $\lim _{n \rightarrow \infty} T x_{n}=\lim _{n \rightarrow \infty} \frac{1}{n}=0$, now, $\lim _{n \rightarrow \infty}\left|B T x_{n}-T B x_{n}\right|=$ $|0-1| \neq 0$. Therefore corollary (3.1) is not applicable.

\section{Competing interests}

The authors declare that they have no competing interests.

\section{Authors' contributions}

All authors have contributed to all parts of the article. All authors read and approved the final manuscript.

\section{References}

[1] Altun, I., H. A. Hancer and Turkoglu, D., A fixed point theorem for multi-maps satisfying an implicit relation on metrically convex metric spaces, Mathematical Communications, 11 (2006), 17-23.

[2] Altun, I. and Turkoglu, D., Some fixed point theorems for weakly compatible mappings satisfying an implicit relation, Taiwanese Journal of Mathematics, 13 (2009), 1291-1304.

[3] Altun, I., Simsek, H., Some fixed point theorems on ordered metric spaces and application, Fixed Point Theory Appl., 2010.

[4] Ali, J. and Imdad, M., An implicit function implies several contraction conditions, Sarajevo J. Math., 4 (17) (2008), $269-285$.

[5] Beg, I. and Butt, A. R., Fixed point for set-valued mappings satisfying an implicit relation in partially ordered metric spaces, Nonlinear Analysis, 71 (2009), 3699-3704.

[6] Berinde, V. and Vetro, F., Common fixed points of mappings satisfying implicit contractive conditions, Fixed Point Theory and Appl., 72 (2010), 4508-4517.

[7] Jungck, G., Compatible mappings and common fixed points, Int. J. Math. Math. Sci., 9 (4) (1986), 771-779.

[8] Jungck, G. and Pathak, H. K., Fixed points via biased maps, Proceedings of the Amer. Math. Soci., 123 (1995), $2049-2059$.

[9] Jungck, G., Rhoades, B. E., Fixed points for set-valued functions without continuity, Ind. J. Pure App. Math. 29 (3) (1998), 227-238.

[10] Jungck, G., Rhoades, B. E., Fixed point theorems for occasionally weakly compatible mappings, Fixed Point Theory, 7 (2006), 287-296. 
[11] Popa, V., Fixed point theorems for implicit contractive mappings, Stud. Cerc. St. Ser. Mat. Univ. Bacau, 7(1997), 127-133.

[12] Popa, V., Some fixed theorems for compatible mappings satisfying an implicit relation, Demonstratio Math., 32(1999), 157-163.

[13] Regan, D. O., Petrusel, A., Fixed point theorems for generalized contractions in ordered metric spaces, J. Math. Anal. Appl., 341 (2008), 1241-1252.

[14] Sharma, S. and Deshpande, B., On compatible mappings satisfying an implicit relation in common fixed point consideration, Tamkang Journal of Mathematics, 33 (2002), 245-252.

[15] Nashine, H. K., and Altun, I., New fixed point results for maps satisfying implicit relations on ordered metric spaces and application, Applied Math. and Computation, 240 (2014), 259-272.

[16] Thagafi, M. A., Shahzad, N., A note on occasionally weakly compatible maps, Int. J. Math. Anal., 3 (2) (2009), 55-58. 